\title{
Patient care: Unmet needs globally
}

\author{
Michael Angastiniotis, Androulla Eleftheriou
}

Thalassaemia International Federation (TIF)

\section{Introduction}

Literature demonstrates that long survival and a good quality of life are achieved where the patients' needs for holistic care are recognised and the appropriate services are offered. The once fatal diseases of childhood have become chronic conditions of adult life. $[8,9,10]$.

TIF's mission is to promote and assist in the implementation of national programmes for the treatment of thalassaemia and other haemoglobin disorders, wherever the patients may be residing, driven by the vision of equal access to quality healthcare for every patient [1].

The purpose of this paper is to report on preliminary results of a global TIF survey that sought to examine the inequalities, which patients experience in their management by services and professionals across the world, and identify some of the reasons contributing to such inequalities. Emphasis in this investigation is given to the services that are offered from the patients' point of view. This work derives from, and is part of TIF's ongoing relationship with its member associations, individual patients, as well as health professional and health authorities.

\section{A framework for mapping and evaluating thalassaemia patients' needs for holistic care}

In an attempt to identify inequalities, we sought for knowledge of the requirements for an optimal system for the care of haemoglobinopathy patients $[2,3,4,5]$. These requirements are dictated by the nature of genetic chronic disorders:

- They are hereditary, and therefore there are implications on pre-conceptual issues, such as parents being aware of their carrier status, since these are Mendelian recessively inherited conditions, prior to the decision to have children. This presupposes a well-informed public, and an adequate counselling system.

- They present in infancy or at the toddler age group and, in the absence of a cure, they must be managed throughout life.

- Vital organs of the body are affected and if not identified early on through a clinical monitoring system, and dealt with according to current evidence-based guidelines, serious and life threatening complications develop.

- Coordinated, multidisciplinary care is a basic requirement, and if provided throughout life, will result in the best possible out-

Correspondence: Michael Angastiniotis.

E-mail: michael.angastiniotis@thalassaemia.org.cy

This work is licensed under a Creative Commons Attribution 4.0 License (by-nc 4.0).

(C) Copyright M. Angastiniotis and A. Eleftheriou, 2018

Licensee PAGEPress, Italy

Thalassemia Reports 2018; 8:7485

doi:10.4081/thal.2018.7485 comes. Results are best if the patients are treated in designated expert centres [6].

From these characteristics, including the pathophysiology, multi-organ involvement and natural history of haemoglobin disorders, certain conditions must be fulfilled to support optimal care, able to achieve the outcomes that are hoped for. These conditions or ingredients can be summarised as follows:

1. Free or affordable healthcare based on country income: Lifelong, expensive treatment with regular blood transfusion, iron chelation medication and involvement of specialised and multidisciplinary services, cannot be sustained by the average family income. This is particularly so in countries with poor economies. In Bangladesh for example, the direct medical costs range from 1632-3960 USD per year, while over $72 \%$ of patients' families have an annual income of 1536-3000 USD, and so cannot possibly provide for their children, while there is no government or other health insurance support. The richest will survive! [11]. The estimated costs are reported quite differently in various countries - in Iran for example, the average annual cost was estimated to be 8322 USD [12] but in this country the governmental support is much higher. Out of pocket expenses can be catastrophic in such chronic conditions as it was demonstrated in a study in China, where $77.7 \%$ of affected families were found to be debt [13]. One possible solution is to achieve universal health coverage, which is the goal of WHO by 2030. The reality, expressed in the 2017 Global Monitoring Report, is that "at least half of the world's population still lacks access to essential health services" and almost 100 million people globally are pushed into extreme poverty each year because of out-of-pocket health expenses [14].

2. Epidemiology-based planning of services: Accurate epidemiological data must be the basis of rational planning of services. Simple questions, such as how many patients with haemoglobin disorders do we have, and how are they distributed across the country, will determine where and how many dedicated centres may be needed. How many reference centres and how these should network with secondary centres is another issue that only epidemiology can answer. If the aim is to provide equal access to effective management then service development can only be based on such facts. Moreover, it should be known whether the number of existing patients is likely to alter over time. In hereditary diseases like the haemoglobin disorders changes will be influenced by the heterozygote population and its marriage patterns. This is true of static populations but the influence of migrations and genetic drift must also be considered. This means epidemiological monitoring and reporting. Haphazard development is uneconomical and will not serve the purpose of equity of care. Epidemiology therefore is required to influence policy and planning, to 'translate' data into actions and interventions. It is from this that the term 'translational epidemiology' is derived [15]. The effective application of epidemiological knowledge however, depends on the accuracy of the data, and this is a major weakness in many countries. The number of patients can only be accurately known if national registries are kept and any changes are regularly monitored, updating the registry. In countries where services are poor, many children die before they are diagnosed and so numbers are underestimated. More often local, clinic based registries are kept in some centres 
and not in others, so national numbers are often 'estimates'. The complications and contribution to premature mortality are also unknown variables.

3. Availability of national policies for the control based on translational epidemiology National policies for the control of haemoglobin disorders, especially policies based on translational epidemiology, are lacking in most countries. Yet without a policy that offers the possibility of reducing birth incidence, and a policy for quality service development, services will be inadequate or ineffective and the objectives of equity and optical care, cannot possibly be achieved. In this respect, epidemiological facts come to support advocacy activities by patient support groups and in this TIF has a responsibility to train and build the capacity of its member associations, to understand and better express the needs of their patients and their families. Policy development is influenced, not only by stakeholder interest, but also by political and social forces, economics, cultural considerations and what are perceived as priorities by current health authorities.

4. Knowledge on prevention and management: All stakeholders involved with haemoglobinopathy patients, professionals, as well as families and the patients themselves, should know the aspects of prevention and management that can result in the best possible outcomes. These elements of holistic management are the results of ongoing research by academic researchers, which indicate the practices that will achieve the desired outcomes. They are published as international guidelines [16, 17], or as national standards, which adapt the guidelines according to national conditions and policies $[18,19]$. The aim is to assist the treating physician by providing decision support aiming to improve the quality of healthcare and to increase patient safety. Optimal, guideline based management has been shown to improve survival [20,21], but also the quality of life of patients [22]. The availability clinical practice guidelines has been upheld as an essential part of quality medicine for decades $[23,24]$ and it is a priority for thalassaemia centres. Many centres complain that optimal care as expressed in TIF guidelines is difficult to follow in economically underprivileged societies. However, this is the reason why associations and federations of associations have been created - to advocate and persuade authorities to aim for the best possible outcomes.

5. Good quality laboratory support: Another service, essential for accurate diagnosis and for monitoring patients is good laboratory support. TIF and other guidelines clearly state the need for diagnostic workup before the first transfusion and they provide a timetable and a list of parameters required for patient monitoring throughout their life $[16,19]$. Such parameters determine treatment decisions and must therefore be followed. Yet thousands of patients are treated according to a $\mathrm{Hb}$ and ferritin check performed at irregular intervals. Proper monitoring will give early warning of complications and has allowed lifesaving interventions [25]. Quality standards for laboratory tests for Haemoglobin disorders have been published [26]. Universal standards for specialised tests such as Magnetic resonance imaging for the quantitation of iron load are still pending in many parts of the world.

6. Continuity of care: Continuity of care from professionals who are experienced in thalassaemia care is still a problem because of staff rotations and inadequate staff training. Expert case management eludes many patients in countries, rich and poor. Professional expertise depends not just on academic qualifications but also on the number of patients treated over the years, adherence to guidelines and good clinical practice. Involvement in research is an added advantage. Networking with other specialists in the field is a major advantage since sharing experiences is one way to assist problem solving in a clinical setting [27]. Networking is also of particular importance in multi-organ conditions such as thalas- saemia and sickle cell disease $[3,5,16]$. Networking is also best facilitated when electronic means are available, since the whole and lifelong picture of each patient can be shared between members of the treatment team.

7. Electronic health records: Electronic health records in the modern world are gaining ground and are most certainly of great importance in lifelong disorders, in which current status can be influenced by past events. Universal adoption of electronic records has yet to be achieved even in developed economically countries. Various barriers are encountered, such as user resistance, variations in local needs, interoperability with existing systems (this is particularly so when disease specific programmes are introduced as additions), system maintenance, governance and others. The need to network in both clinical practice and research, also brings technical issues, like interoperability, to the forefront, as well as issues of data protection and confidentiality. None of these issues are, however, insurmountable obstacles and when the benefits of information technology versus piles of paper records, are realised then the electronic health record is realised as a necessity. Such a system allows a true picture of trends over time and a true clinical picture of individual patients or groups of patients, and especially the outcomes.

8. Patient participation in care, service provision and research: Patient input in all aspects of care, service provision and research is essential. Much has been written about the 'partnership' versus the 'paternalistic' approach to patient care, its effect on adherence to treatment, self-management and the overall psychological health of the patient, especially where chronic diseases are concerned [28, 29 $30,31]$. Basic to partnership is the doctor/patient relationship but none of this can be achieved without patients being well informed on their condition and its management. Patient information is a duty of the treating physician and his team. Patient education has been a principal advocated by TIF from its foundation as evidenced by its publications [32], the online patient educational platform (launched 2017) and its regional and international conferences which are designed for patient involvement. Patients cannot be passive recipients of treatment but active participants in interventions that affect their everyday life and their survival.

Availability and Quality of holistic services is the central concept of the elements described above. Quality assessment for the purpose of accreditation, involves much more and TIF has embarked on a programme that seeks to recognise and accredit centres of excellence for haemoglobin disorders, which is hoped to encourage more centres to reach a level of patient-centred care that will achieve outcomes that are expected, according to the achievements of medical science in the $21^{\text {st }}$ century, and keep up with progress and new developments.

As a patient driven organisation, TIF has been monitoring patient concerns and has been encouraging them to voice these concerns during conferences as well as to their national health authorities. Examples of these concerns taken from patient quotes, are listed in Table 1.

From reports and from local visits, experts are aware that many elements of optimal care are missing in many centres. Examples include the non-existence of guidelines or standards for patient care and adherence to them is poor, outcomes such as survival rates, complication rates are not recorded and in general the average age of patients reflects poor results, adherence to treatment is poor, social achievements (education, marriage, employment, integration) are often also poor reflecting lack psychosocial support. Out of pocket expenses are the rule in many countries $[13,14]$.

In order to estimate the level of services as perceived by patients, TIF has conducted a survey among thalassaemia patients globally. At the time of writing, not all the results were available but a preliminary report is presented here. 


\section{Methodology}

\section{Design}

As mentioned earlier, to map the necessary services to constitute a holistic patient care and assess the extent to which these services are available at good quality level to patients across affected countries, we drew on the following 8 categories to formulate questions that could elicit patients' evaluations.

1. Free or affordable healthcare based on country income

2. Epidemiology-based planning of services

3. Availability of national policies for the control based on translational epidemiology

4. Knowledge on prevention and management

5. Good laboratory support

6. Continuity of care

7. Electronic health records

8. Patient participation in care, service provision and research:

The questionnaire was directed to patients and families of patients with thalassaemia of various varying severity forms including multi-transfused beta thalassaemia patients (name the forms included in questionnaire).

The questionnaire was distributed online via the Survey monkey software and supplemented by hard copies for patients who did not have access to internet connection.

\section{Analysis}

The Human Development Index (HDI) was chosen as a valuable resource in the analysis of the data and interpretation of results and relevant to the purposes of this research to highlight the relationship between the level and quality healthcare services for thalassaemia patients, and promote policies and healthcare systems for amendment.

The Human Development Index (HDI) is a summary measure of average achievement in key dimensions of human development: a long and healthy life, being knowledgeable and have a decent standard of living. The HDI is the geometric mean of normalized indices for each of the three dimensions. The Human Development Index (HDI) was created by the United Nations Development Programme (UNDP) to emphasize that people and their capabilities should be the ultimate criteria for assessing the development of a country, not economic growth alone. The HDI can also be used to question national policy choices, asking how two countries with the same level of GNI per capita can end up with different human development outcomes. These contrasts can stimulate debate about government policy priorities. In this preliminary report, a total of 106 online responses were analysed. The majority of responses were from adult university graduates, which means that whichever country they come from, they were the best examples of the patient population, not reflecting the total population of patients especially in the countries which have not achieved a very high Human Development Index (HDI) [33].
The average age of this patient group was 29.8 years (range 2 to 57 years). Those over 20 who responded to questions relevant to adults, had an average age of 34.2 years.

\section{Results}

\section{Demographics}

The 106 respondents were from 33 countries:

\begin{tabular}{lll}
\hline Who region & & Number of patients \\
\hline Europe & 11 countries & 24 patients \\
\hline East Mediterranean & 5 countries & 17 patients \\
\hline South East Asia & 7 countries & 35 patients \\
\hline West Pacific & 4 countries & 7 patients \\
\hline Americas & 4 countries & 21 patients \\
\hline Afro & 2 countries & 2 patients \\
\hline TOTAL & 33 countries & 106 patients \\
\hline
\end{tabular}

These countries were classified according to the Human Development Index (HDI) and the responses were classified according to the HDI of the country of residence of the patient.

Seven indicators were chosen for this preliminary study to establish patients' view of the quality of services:

1. The pre-transfusion haemoglobin level at which they are transfused

2. The pre-transfusion haemoglobin, in relation to the delays in blood supplies

3. The out-of-pocket expenses for the provision of essential treatment

4. The frequency of MRI T2* estimation of iron load in the heart

5. The marital status of adult patients

6. The employment status of qualified patients

7. Patients' confidence in the correctness of their treatment.

1. Pre-transfusion haemoglobin should be, according to agreed guidelines [16], 9-10.5g/dl. In the responses to this survey, those that receive blood transfusions according to the guidelines are mostly form very high HDI countries, as shown in Figure 1. While those transfused at $\mathrm{Hb}$ levels below $9 \mathrm{~g} / \mathrm{dl}$ are from high and medium to low HDI countries.

2. The presence of delays in providing blood at the right time for each patient in order to maintain a good level of $\mathrm{Hb}$, reflect the level of efficiency and effectiveness of blood donation drives, but it is also an indirect indicator of the quality of the blood transfusion services of a country and the community involvement. Again, our results demonstrate that it is patients in medium/low HDI countries who experience delays and so have to live with lower $\mathrm{Hb}$ levels (with all the consequences of poor oxygenation and extramedullary erythropoiesis). Figure 2.

3. Sustained provision of care is impossible for many families

\section{Table 1. Patient comments on services that they want.}

- "Medical \& nursing staff that I can trust"
- Good communication: given TIME and staff that LISTENS
- Consideration for the patients' needs for a normal life (clinic times, waiting time): fitting treatment around the patients' life and not administration needs.
- Knowing that protocols and standards of care are followed
- Patient involvement in planning services \& setting standards
- Ensuring that patient reported outcomes are recorded and considered


due to the high out-of-pocket expenses required. Patients' responses to our survey indicate that full state coverage is available to less than half of the patients around the world who answered our questionnaire (48.7\%). The degree of coverage by various insurance schemes was not made clear, but it is felt that it is not likely that all aspects of chronic care is covered. This goes to suggest that half of thalassaemia patients require family assistance to maintain optimal care. Figure 3.

4. Patients are recommended to have an MRI test for cardiac iron on an annual basis. In this survey, $60 \%$ of patients from medium/low HDI countries have never had this test and this includes over a third of patients from high HDI countries. Figure 4.

5. Marital status reflects more than the patients' health, but also several social and cultural issues (such as prejudice), in which economic factors may play a minor role. In patients over 20 years old, in this patient population the cultural background was considered: Table 2 .

6. The employment status of the patient respondents in this survey reflects their characteristics since most were educated and university graduates. In several countries, factors such employer prejudice, economic factors but also the family's and patients' view of the ability to work may play a significant role. Figure 5 shows that for qualified adult thalassaemia patients, the HDI status of the country does not significantly affect the chances of employment and a significant number of patients across the globe stay away from useful employment by choice.

7. Patients' confidence in the correctness of their treatment can be taken as indicator of the sufficiency and quality of communi-

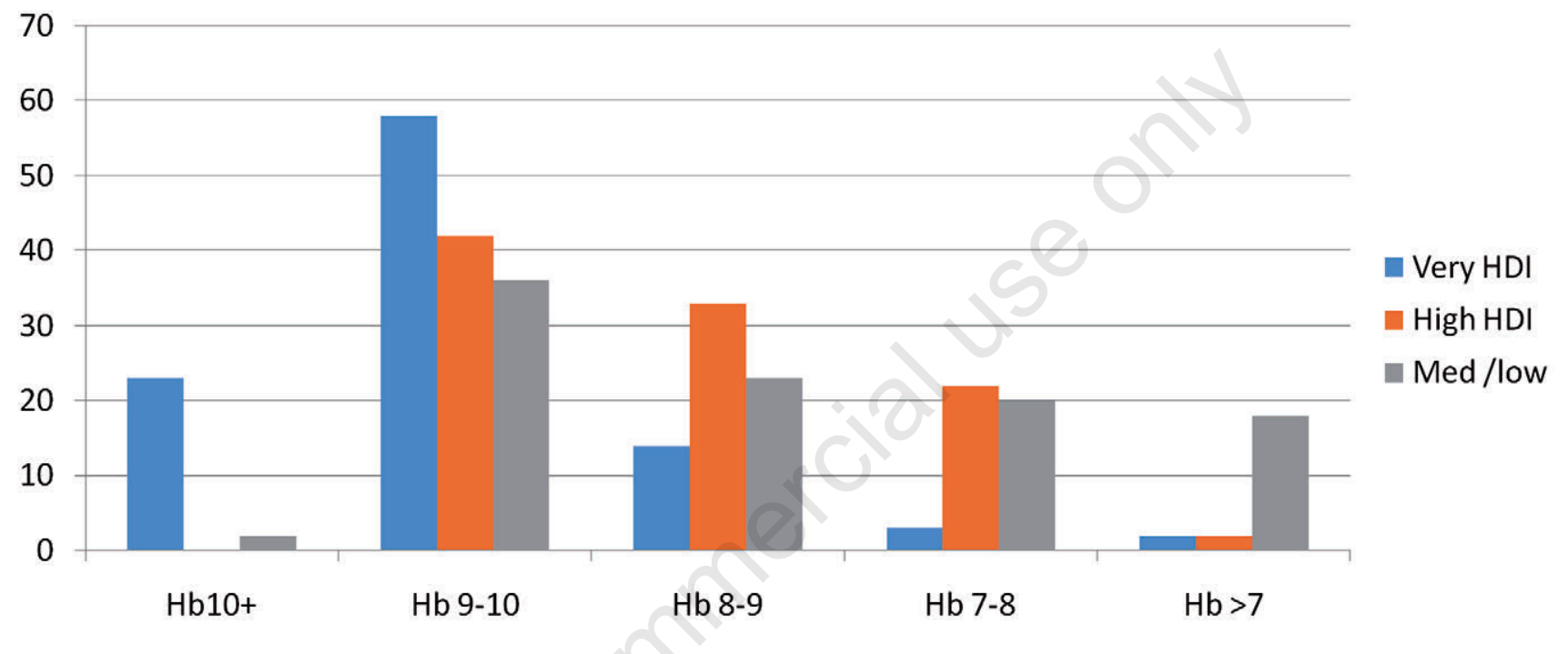

Figure 1. Pre-transfusion haemoglobin levels according to country HDI level.

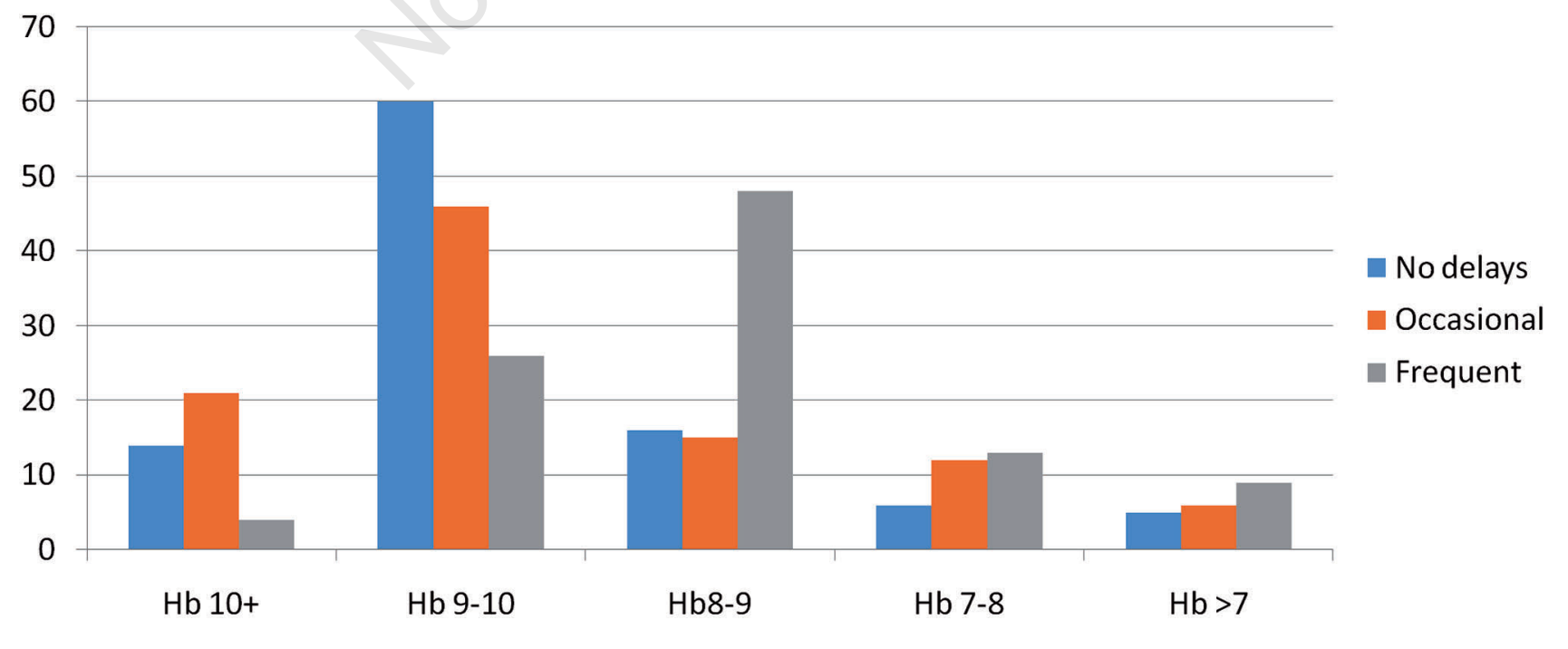

Figure 2. Classification of delays with respect to country HDI. 
cation between patients and their treating physicians, a key service of healthcare directly related to patient's right to information (Figure 6).

- $47 \%$ are uncertain about the correctness (half of these are sure that treatment is not correct).

- Patients from all centres gave similar responses.

- Similar responses in a European survey 2014.

\section{Discussion and Conclusions}

To assess patients' views several more indicators have been introduced into the questionnaire, but were not assessed in this preliminary analysis, which is inevitably limited (The questionnaire can be viewed at its entirety at https://goo.gl/vNkxud). Not least, the data reported herein are patient reported outcomes, which essentially reflect their expectations as well as the factors that they perceive affect their quality of life. Other questions that are included are clinic working hours, transfusion timetables, days lost from work and school due to treatment and many others which can affect patient satisfaction. This paper is intended as an introduction to a more extensive study, but also a general indicator of issues affecting quality of care. The patients' view is very rarely studied and published. In this survey, the common denominator is a patients group of the same diagnostic category even though from many different backgrounds. Comparisons in such a heterogeneous group can only be made if they are categorised into groups with similar characteristics and the common denominator chosen in this case is the Human Development Index.

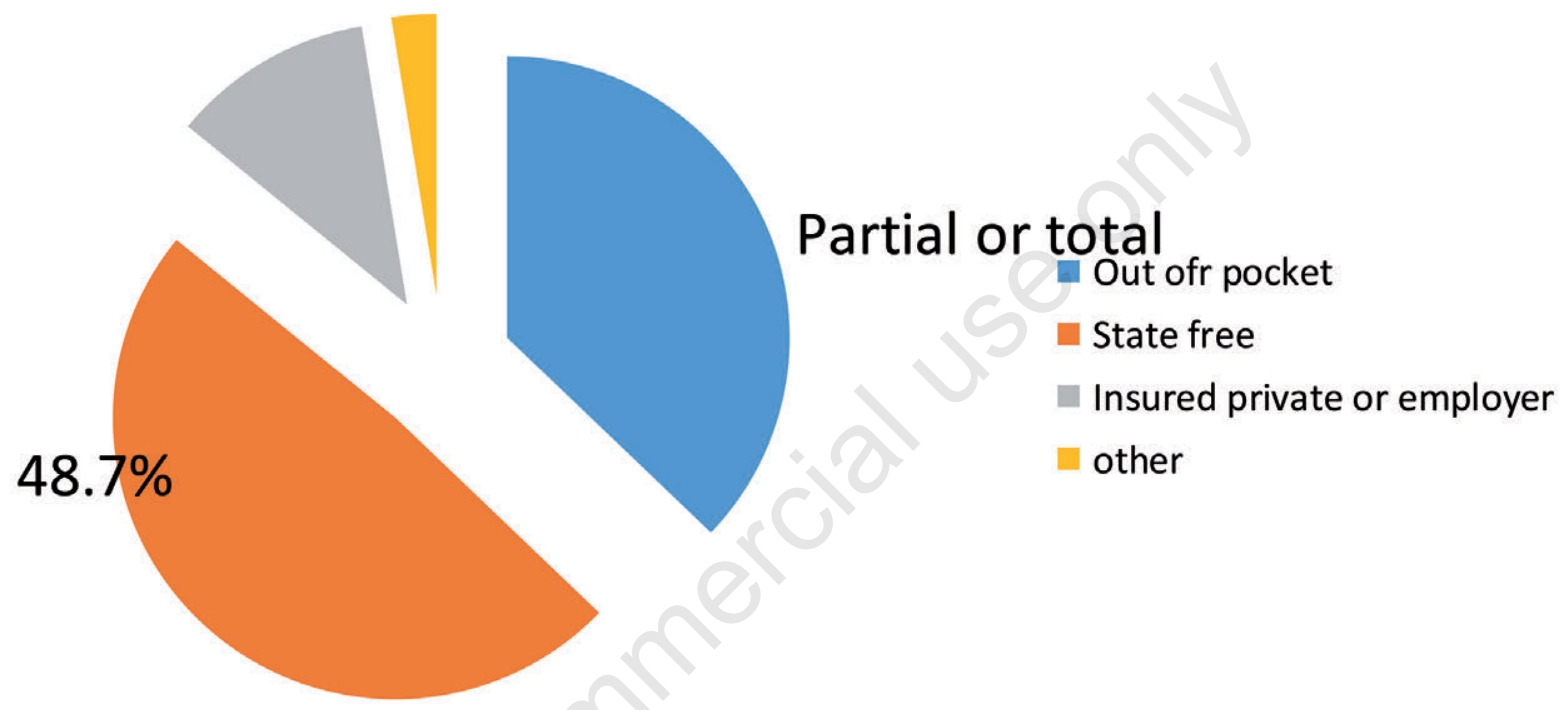

Figure 3. Availability of partial or total healthcare coverage according to country HDI classification.

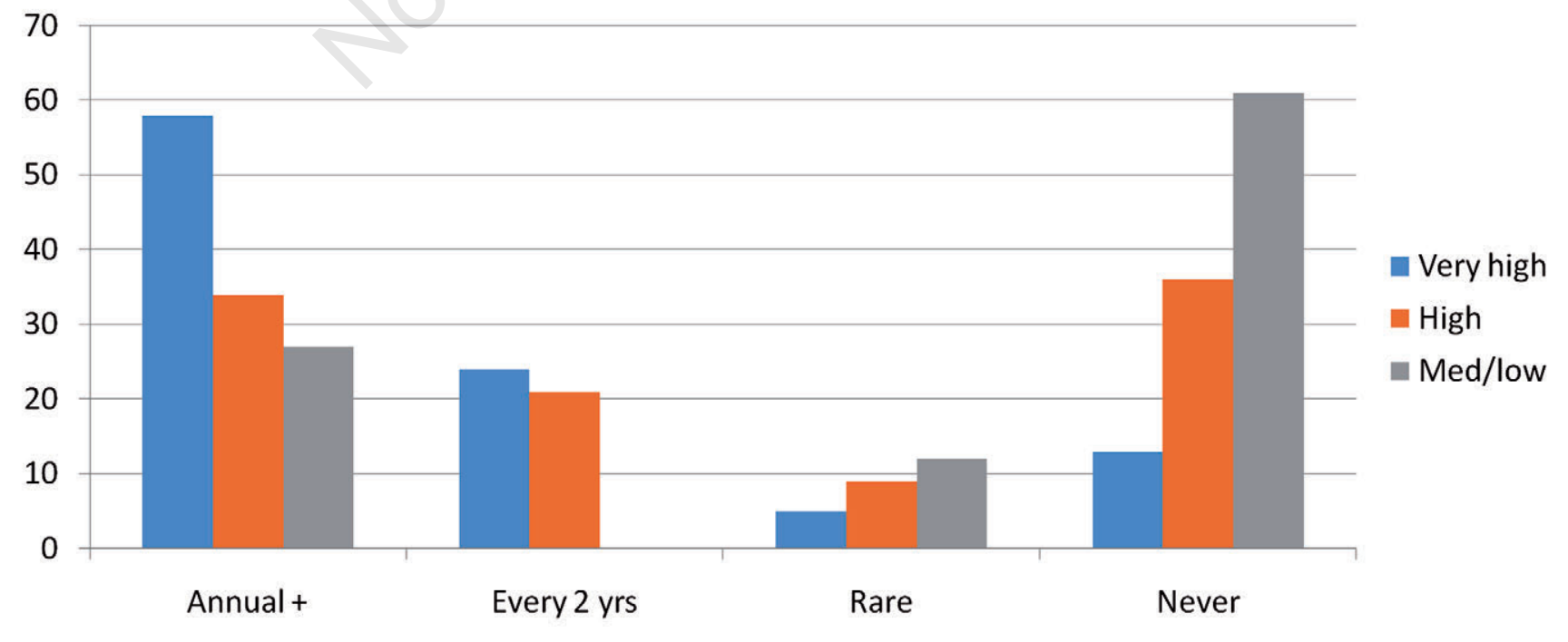

Figure 4. Frequency of MRI testing for cardiac iron and country HDI classification. 
The main weakness of this survey is the small sample size made availablefor each HDI category; nevertheless, certain interesting conclusions can be drawn:

- Concerning pre-transfusion $\mathrm{Hb}$ level, it is noteworthy that around $15 \%$ of patients living and being treated in very high HDI countries state they are receiving transfusions below the recommended range. The reasons cannot be discerned from this study, and there may be acceptable clinical reasons. However, ignorance of guidelines and patient non- compliance in some centres may be factors and a more in-depth analysis is needed. The fact that $60 \%$ to $65 \%$ of patients in high, medium and low HDI countries are transfused below recommended levels is not surprising but it does signal a need for more vigorous professional education, advocacy for policy changes and patient education.

- Frequent delays in providing blood, a reflection on the blood banking system and the donation campaigns is again a characteristic more of low-income countries. This is not surprising, since according to the WHO, the highest percentage of blood donations take place in HDI countries. For example, the European WHO region, inhabited by $11 \%$ of the global population [34] and home to only $3.3 \%$ of the world's known thalassaemia population [35], reported $30 \%$ of the global blood donations.

- Behind much of the suffering and poor service provision in many countries is the lack of policies to support patients and their families, enabling them to provide even basic elements of management. Even blood and essential drugs have to be paid for and in chronic diseases, this leads to catastrophic results and early loss of patients. This is a major global issue in the WHO's sustainable development goals [36]. Unless governments and policy makers resolve this issue, thalassaemia patients will continue to miss the kind of treatment that can guarantee a prolonged life span that so many other patients are enjoying, even within the same country. Employment and marriage are aspects of life that are the results of many factors medical and social factors, but again inequalities are evident even in developed economies.

- One major issue is the professional-patient relationship. In this preliminary paper only one issue was discussed, that of trust in the treatment that is provided. In this aspect, the results from this survey were compared to a similar survey conducted by TIF within a project called ENERCA in 2012-14, in which the participants were European patients [37]. The results are very similar in that only $57 \%$ of the total patients asked felt that their treatment was correct while the rest were unsure. This issue of patient trust to their treating physicians' choices in their treatment, along with poor adherence to guidelines by centres and limited financial support seem to be the most serious contributors to poor outcomes.

It is important to note that, this survey represents patients' perceptions regarding the treatment they receive, a perspective seldom reported in the literature on haemoglobinopathies and one that merits more careful and more frequent evaluation. . It would be useful to correlate and contrast these results with input from professionals in these countries to enhance the validity of our conclusions.

Table 2. Marital status of thalassaemia patients across cultures.

\begin{tabular}{lccc} 
Cultural group & Married patients & Single & Cohabiting \\
Western culture & $48 \%$ & $34 \%$ & $13 \%$ \\
Arab culture & $35 \%$ & $60 \%$ & 1 case \\
\hline Asian populations & $20 \%$ & $78 \%$ & 0 \\
\hline
\end{tabular}

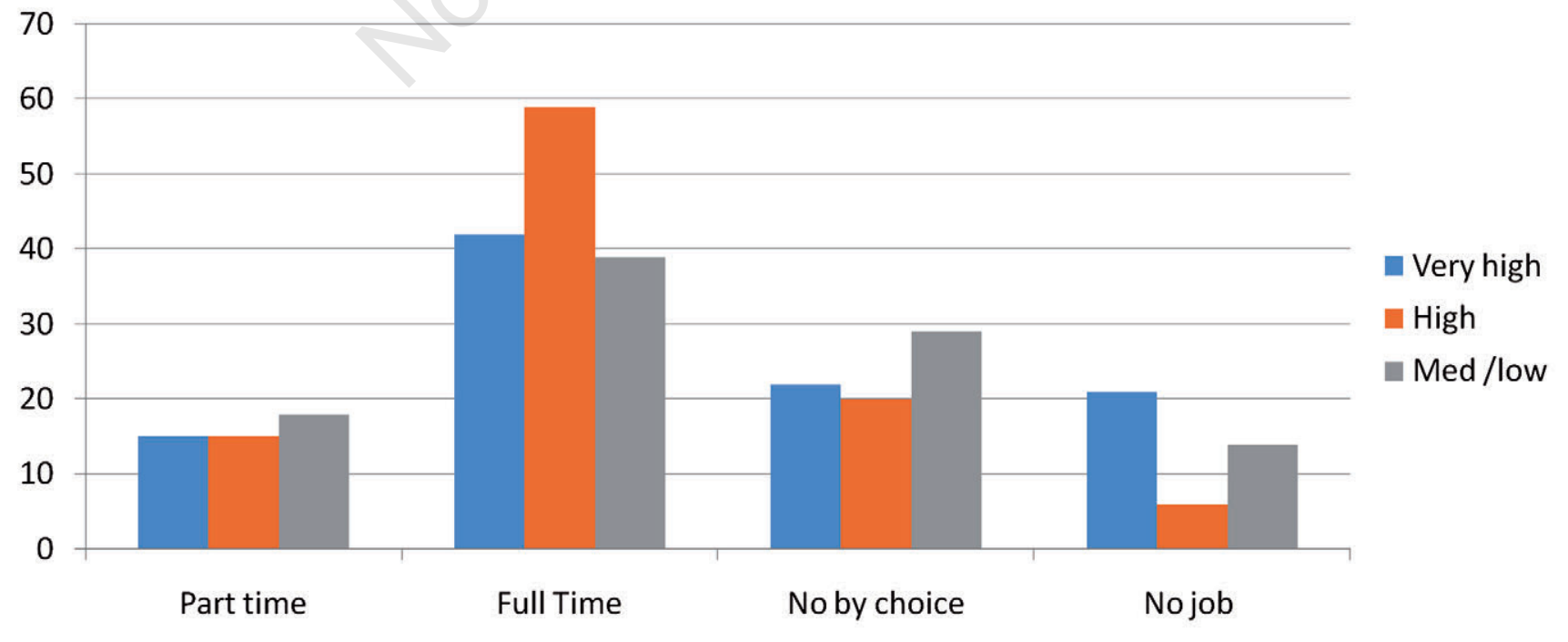

Figure 5. Employment status of thalassaemia patients according to country HDI classification. 


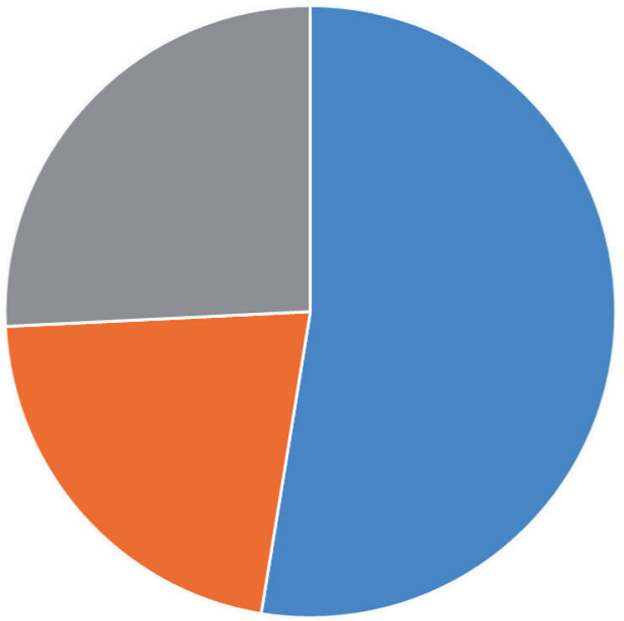

treatment is correct $\quad$ Not correct $\quad$ Not sure

\section{Figure 6.}

\section{References}

1. Thalassaemia International Federation (2018) TIF's mission and vision. Available atwww.thalassaemia.org.cy

2. Tubman VN, Fung EB, Vogiatzi M, Thomson AA et al. Guidelines for the standard monitoring of patients with thalassaemia: report of the Thalassaemia Longitudinal Cohort. J Paediatr Hematol Oncol. 2015; 37(3): e162-e169.

3. Origa R, Baldan A, Marsella M, Borgna-Pignatti C. A complicated disease: what can be done to manage thalassaemia major more effectively? Expert Rev Hematol. 2015; 8(6): 851-62.

4. Rund D. Thalassaemia 2106: modern medicine battles with an ancient disease. Am J Hematol. 2016; 91(1): 15-21.

5. Origa R. $\beta$-thalassaemia. Genet Med 2017; 19(6): 609-619.

6. Taher AT, Weatherall DJ, Cappellini MD. Thalassaemia. Lancet. 2017; pii: S0140-6736(17)31822-6. doi: 10.1016/ S0140-6736(17)31822-6. Review.

7. Forni GL, Puntoni M, Boeri E, Terenzani L, Balocco M. The influence of treatment in specialized centers on survival of patients with thalassemia major. Am J Hematol. 2009; 84(5):317-8.

8. Telfer P, Coen PG, Christou S, Hadjigavriel M, Kolnakou A, et al. Survival of medically treated thalassaemia in Cyprus. Trends and risk factors over the period 1980-2004. Haematologica. 2006; 91: 1187-92.

9. Borgna-Pignatti C. The life of patients with thalassaemia major. Haematologica. 2010; 95(3): 345-348.

10. Ladis V, Chouliaras G, Berdoukas V, Chatziliami A, Fragodimitri C et al. Survival in a large cohort of Greek patients with transfusion dependent thalassaemia and mortality ratios compared to the general population. Europ J Haematol. 2011; 86: 332-338.

11. Hossain MS, Raheem E, Sultana TA, Ferdous S, Nahar N et al. Thalassaemias in South Asia: clinical lessons learnt from Bangladesh. Orphanet J Rare Dis. 2017;12(1):93.

12. Esmaeilzadeh F, Azarkeivan A, Emamgholipour S, Sari AA et al. Economic burden of thalassaemia major in Iran. J Res Health Sci. 2016 Summer; 16(3):111-115.
13. Blue Book of Thalassaemia in China. AngelMom Charity foundation and the China Philanthropy research Institute. 2016.

14. Tracking Universal Health Coverage: 2017 Global Monitoring Report. World health Organisation \& World Bank.

15. Marks JS. Epidemiology, public health and public policy. Prev Chronic Dis. 2009; 6(4): http://www.cdc.gov.pcd/issues/ 2009/oct/09_0110.htm.

16. Cappellini MD, Cohen A, Porter J, Taher A, Viprakasit V et al. Guidelines for the Clinical Management of Transfusion Dependent Thalassaemia. 3rd Edition 2014; Thalassaemia International Federation publication 20.

17. Guidelines for the Management of Non transfusion Dependent Thalassaemia [NTDT]. 2nd Edition. 2017. Thalassaemia International Federation publication 22.

18. Vichinsly E, Levine L, Bhatia S, Bojanowski J, Coates T, Foote D, Fung E, et al. Standards of Care Guidelines for Thalassemia 2012. Children's' Hospital and Research Center Oakland.

19. Yardumian A, Telfer P, Shah F, Ryan K, Darlison MW, BA MA PhD, Miller E, Constantinou G, et al. Standards for the Clinical Care of Children and Adults with Thalassaemia in the UK. 3rd Edition, 2016. United Kingdom Thalassaemia Society.

20. Telfer P, Coen PG, Christou S, Hadjigavriel M, Kolnakou A, et al. Survival of medically treated thalassaemia in Cyprus. Trends and risk factors over the period 1980-2004. Haematologica. 2006; 91: 1187-92.

21. Voskaridou E, Ladis V, Kattamis A, Hassapopoulou E, Economou M, Kourakli A, et al. A national registry of haemoglobinopathies in Greece: deducted demographics, trends in mortality and affected births. Ann Hematol. 2012; 91(9): 1451-8.

22. Ali SS, Tarawah AM, Al-Hawsawi ZM, Zolaly MA, Turkustani W. Comprehensive patient care improves the quality of life in transfusion dependent patients with $\beta$-thalassaemia. Saudi Med J. 2015; 36(5): 575-579.

23. Kredo T, Bernhardsson S, Machingaidze S, Young T, Louw Q, Ochodo E, Grimmer K. Guide to clinical guidelines: the current state of play. Int J for Quality in health care. 2016;28(!): $122-128$

24. Mussalam KM, Angastiniotis M, Elefhteriou A, Porter JB. Cross-talk between available guidelines for the management of patients with beta-thalassaemia major. Acta Hematol. 2013; 130: 64-73

25. Modell $\mathrm{B}$, Khan $\mathrm{M}$, Darlison $\mathrm{M}$, Westwood MA, Ingram $\mathrm{D}$, Pennell DJ. Improved survival of thalassaemia major in the UK and relation to $\mathrm{T} 2 *$ cardiovascular magnetic resonance. J Cardiovasc Magn Reson. 2008;10:42. doi: 10.1186/1532429X-10-42.

26. Traeger-Synodinos J, Harteveld CL, Old JM, Petrou M, Galanello R, Giordano P, Angastioniotis M, De la Salle B, Henderson S, May A. EMQN Best Practice Guidelines for molecular and haematology methods for carrier identification and prenatal diagnosis of the haemoglobinopathies. . EMQN haemoglobinopathies best practice meeting. Eur J Hum Genet. 2015 Apr;23(4):426-37.

27. Angastiniotis M, Eleftheriou A. Requirements for a reference or expert thalassemia center: the structure/model for centers dealing with chronic/hereditary blood disorders. Hemoglobin. 2009;33 Suppl 1:S204-10.

28. Wagner EH, Bennett SM, Austin BT, Greene SM, Schaefer JK, Vonkorff M. Finding common ground: patient-centeredness and evidence-based chronic illness care. J Altern Complement Med. 2005: 11 Suppl 1: S7-15. 
29. Hudon C, Fortin M, Haggerty J, Loignon C, Lambert M, Poitras ME. Patient-centred care in chronic disease management: a thematic analysis of the literature in family medicine. Patient Educ Couns. 2012; 88(2): 170-6.

30. Shamsi A, Amiri F, Ebadi A, Ghaderi M. The effect of partnership care model on mental health of patients with thalassaemia major. Depress Res Treat. 2017; 3685402.

31. Kaye J, Curren L, Anderson N, Edwards K, Fullerton SM, Kanellopoulou $\mathrm{N}$ et al. From patients to partners: participant- centric initiatives in biomedical research. Nat Rev Genet. 2012 Apr 3;13(5):371-6.

32. Eleftheriou A, About thalassaemia. TIF publication 2003.

33. hdr.undp.org/en/content/human-development-index-hdi

34. 2016 Global Status report on blood safety and availability. WHO http://apps.who.int/iris/bitstream/10665/254987/1/ 9789241565431-eng.pdf

35. TIF Global database (unpublished).

36. http://www.who.int/health_financing/documents/en/ 